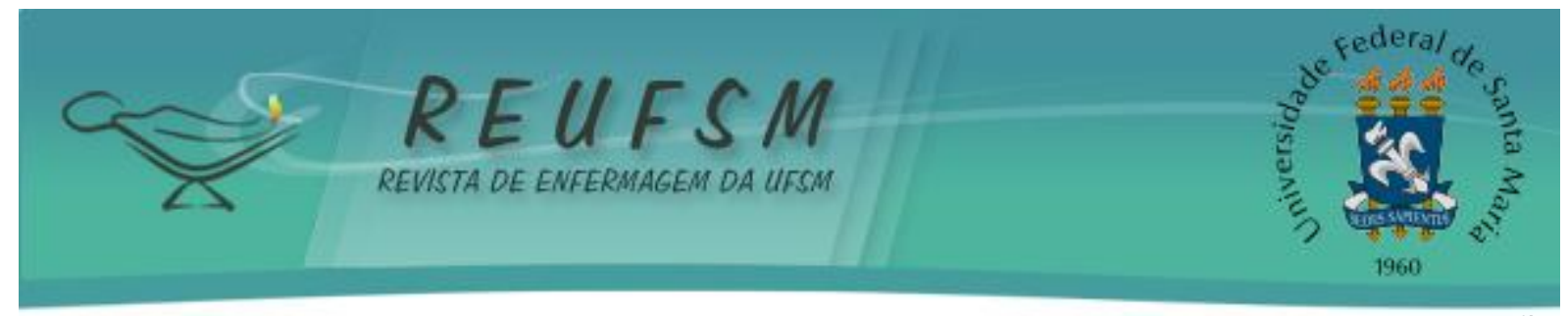

ARTIGO DE REVISÃO

\title{
MATUTINO, VESPERTINO OU INDIFERENTE? PRODUÇÃO DO CONHECIMENTO SOBRE O CRONOTIPO NA ENFERMAGEM
}

\author{
MORNING, EVENING OR INDIFFERENT? PRODUCTION OF KNOWLEDGE ON CHRONOTYPE \\ IN NURSING
}

\section{MATUTINO, VESPERTINO O INDIFERENTE? PRODUCCIÓN DE CONOCIMIENTO SOBRE CRONOTIPO EN ENFERMERÍA}

\author{
Rosângela Marion da Silva ${ }^{1}$ \\ Regina Célia Gollner Zeitoune ${ }^{2}$ \\ Carmem Lúcia Colomé Beck ${ }^{3}$ \\ Marli Maria Loro ${ }^{4}$
}

Doi: $10.5902 / 2179769212888$

RESUMO: Objetivo: analisar o acervo científico sobre o cronotipo na enfermagem. Método: revisão integrativa que teve como questão norteadora: Qual a produção do conhecimento sobre o cronotipo na enfermagem? Foi realizada nas bases de dados LILACS, Pubmed e biblioteca virtual SCIELO. Incluiram-se artigos originais publicados entre os meses de janeiro de 2000 e dezembro de 2013. Resultados: 14 artigos foram considerados elegíveis para análise, os quais tratam da (dis) concordância entre o cronotipo e turno de trabalho, e da associação do cronotipo com variáveis. A maioria das pesquisas identificou o cronotipo indiferente entre os participantes. Conclusão: alocar o trabalhador em turno condizente ao cronotipo pode repercutir na qualidade de vida e do cuidado. Sendo assim, são necessárias investigações com outras variáveis com o objetivo de identificar se a discordância entre cronotipo e turno de trabalho associa-se a maiores repercussões na saúde do trabalhador.

Descritores: Enfermagem; Trabalho em turnos; Ritmo circadiano.

ABSTRACT: Objective: to analyze the scientific literature on chronotype in nursing. Method: integrative review guided by the question: What is the production of knowledge about chronotype in nursing? The review was performed in the databases LILACS, PubMed and the electronic library SCIELO. It included original articles published between the months of January 2000 and December 2013. Results: 14 articles were considered eligible for analysis, which address the (dis) agreement between chronotype and the work shift, and the association of variables with chronotype. In most studies, the indifferent chronotype was identified among the participants. Conclusion: setting the worker's shift according to their chronotype can influence the quality of life and care. Thus, investigations with other variables are necessary in order to identify if the disagreement between chronotype and the work shift is associated with a greater impact on workers' health.

\footnotetext{
${ }^{1}$ Enfermeira. Doutora em Ciências. Professor Adjunto A do Departamento de Enfermagem da Universidade Federal de Santa Maria (UFSM). Santa Maria, Rio Grande do Sul, Brasil. E-mail: cucasma@terra.com.br ${ }^{2}$ Enfermeira. Doutora em Enfermagem. Professora Titular do Departamento de Enfermagem de Saúde Pública da Escola de Enfermagem Anna Nery da Universidade Federal do Rio de Janeiro (UFRJ). Rio de Janeiro, Brasil. E-mail: regina.zeitoune@gmail.com

${ }^{3}$ Enfermeira. Doutora em Filosofia da Enfermagem. Professora Associado do Departamento de Enfermagem da Universidade Federal de Santa Maria (UFSM). Santa Maria. Rio Grande do Sul, Brasil. E-mail: carmembeck@gmail.com

${ }^{4}$ Enfermeira. Doutora em Ciências. Professor de Enfermagem da Universidade Regional do Noroeste do estado do Rio Grande do Sul (UNIJUI). Santa Maria, Rio Grande do Sul, Brasil. E-mail: marlil@unijui.edu.com
} 


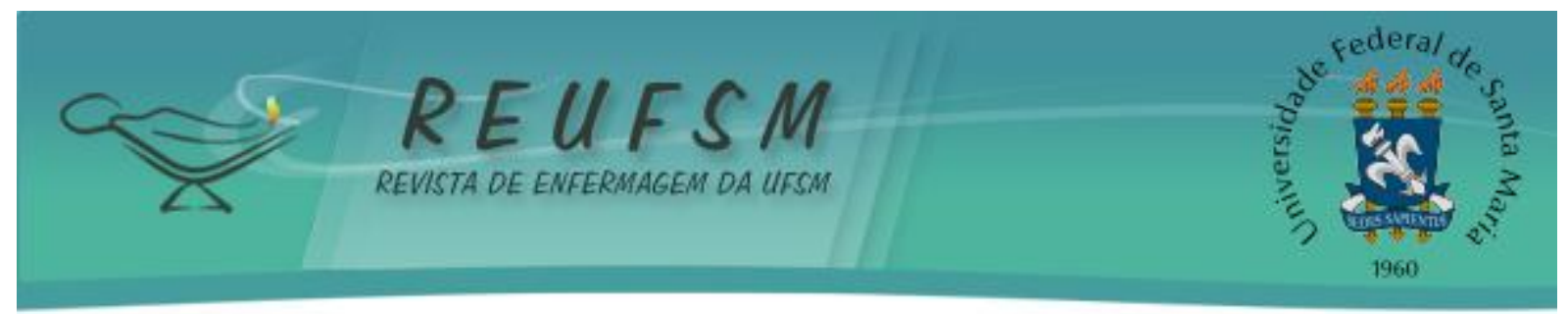

Descriptors: Nursing; Shift work; Circadian rhythm.

RESUMEN: Objetivo: analizar la evidencia científica sobre el cronotipo en enfermería. Método: revisión integradora cuya pregunta orientadora fue: ¿Cuál es la producción de conocimiento sobre cronotipo en enfermería? Se realizó en las bases de datos LILACS, PubMed y biblioteca virtual Scielo. Se incluyeron artículos originales publicados entre los meses de enero/2000 y diciembre/2013.Resultados: 14 artículos fueron considerados elegibles para el análisis, que tratan el (des) acuerdo entre cronotipo y el trabajo por turnos, y la asociación de variables con cronotipo. La mayoría de investigaciones identificó el cronotipo como indiferente para los participantes. Conclusión: asignar al trabajador acorde con el cronotipo puede influir en la calidad de vida y la atención. Por tanto, son necesarias investigaciones con otras variables con el fin de identificar si la discrepancia entre cronotipo y turno de trabajo se asocia a un mayor impacto en la salud del trabajador.

Descriptores: Enfermería; Trabajo por turnos; Ritmo circadiano.

\section{INTRODUÇÃO}

0 processo de trabalho na enfermagem é singular, determinado pela realização do trabalho em turnos com o objetivo de prestar assistência contínua à população. ${ }^{1-2}$ Essa organização em turnos predispõe o trabalhador a alterações na saúde uma vez que, dependendo do cronotipo e turno de trabalho, pode haver uma inversão no ciclo vigíliasono, o que exige do organismo esforços para adaptar-se a condição imposta.

Nesse contexto é necessário considerar a preferência individual dos trabalhadores pelo momento de melhor desempenho para as atividades e para o descanso, denominada cronotipo, com vistas a minimizar possíveis danos à saúde relacionados ao trabalho. Cronotipo é uma tendência individual pela escolha do momento em realizar atividades e pelo descanso, refletindo na capacidade para dormir cedo ou tarde da noite e acordar cedo ou tarde. ${ }^{3}$

0 cronotipo pode ser classificado em três tipos considerando-se as diferenças individuais de adaptação temporal dos ritmos biológicos. O tipo matutino (dividido em tipos moderado e extremo) é característico de indivíduos que preferem dormir cedo e também acordar cedo sem dificuldades, estando já nesse momento perfeitamente aptos para o trabalho e apresentando bom nível de alerta, desempenho físico e mental pela manhã. Os indivíduos do tipo vespertino (dividido em tipos moderado e extremo) preferem dormir e acordar tarde, com melhor disposição e desempenho no período da tarde e início da noite. E tipo indiferente, peculiar de indivíduos que têm maior flexibilidade, que escolhem horários intermediários de acordo com as necessidades de sua rotina. ${ }^{4}$

No contexto do trabalho, é importante considerar a singularidade do trabalhador e a sua disposição em determinado horário para realizar as atividades. Assim, considerando a necessidade de aprofundar o debate que envolve o estudo referente à saúde do trabalhador que atua em turnos, em especial os da enfermagem, este estudo tem como objetivo analisar o acervo científico sobre o cronotipo na enfermagem.

\section{MÉTODO}

Pesquisa em base documental, fundamentada em pressupostos da revisão integrativa de literatura, método que permite incluir estudos com diferentes abordagens metodológicas e que agrupa resultados de um conjunto de pesquisas com temáticas semelhantes. $^{5}$ Esse tipo de pesquisa tem sido utilizada na enfermagem para o 


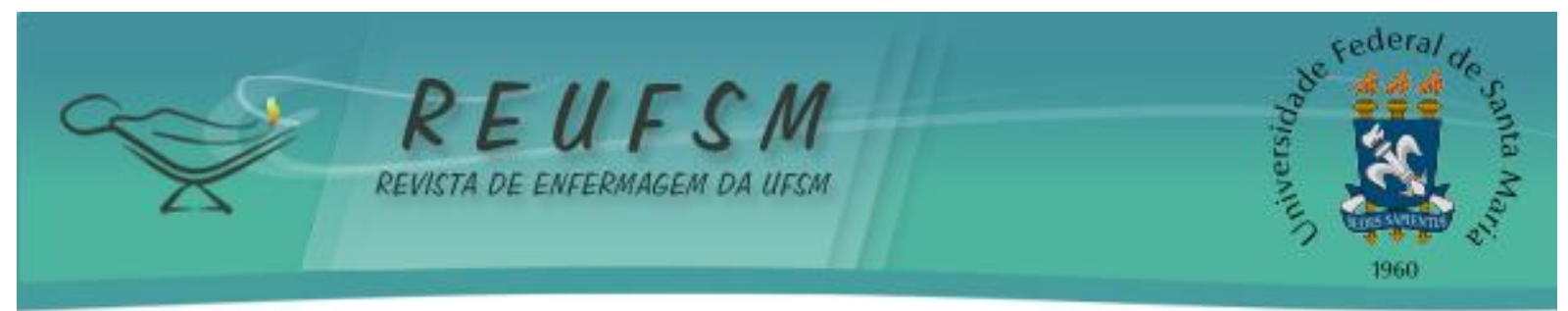

aprofundamento de assuntos por parte dos profissionais, e essa iniciativa contribui para o desenvolvimento da prática da enfermagem baseada em evidências. ${ }^{6}$

Foi direcionada pelas seguintes etapas: formulação da questão norteadora da revisão, estabelecimento de critérios para a inclusão e exclusão dos estudos, categorização e avaliação dos estudos incluídos, discussão e interpretação dos resultados e a síntese do conhecimento evidenciado nos artigos analisados. ${ }^{5}$ A questão norteadora foi: Qual a produção do conhecimento sobre o cronotipo na enfermagem entre 2000 e 2013?

Utilizou-se a palavra chave cronotipo/chronotype e os descritores do Descritores em Ciências da Saúde (DECS) e do Medical Subject Headings (MeSH) em diferentes combinações: trabalho em turnos/shift work, trabalho noturno/night work, ritmo circadiano/circadian rhythm, enfermagem/nursing. Esses termos foram pesquisados nas bases de dados Literatura Latino-Americana e do Caribe em Ciências da Saúde (LILACS), Public Medline (PubMed), e na biblioteca virtual Scientific Electronic Library Online (SCIELO). Para ser incluído nessa revisão o estudo deveria ser pesquisa original, ter sido publicado entre janeiro de 2000 e dezembro de 2013, ter sido realizado com a enfermagem e estar escrito em inglês, português ou espanhol. Foram excluídos estudos repetidos nas bases de dados, dissertações e teses, revisões de literatura e protocolos de pesquisa.

A coleta dos dados ocorreu em novembro 2013, reaplicada em janeiro de 2014. Para os artigos que não estavam disponíveis nas bases de dados, realizou-se o contato com a revista em que o artigo foi publicado ou com os autores do artigo solicitando a disponibilidade do documento. Sobre a extração dos dados, inicialmente foi elaborado um instrumento sistematizado, o que possibilitou identificar em cada estudo o objetivo, método utilizado e principais conclusões. $\mathrm{Na}$ análise e interpretação dos dados, as informações extraídas foram comparadas e os conteúdos agrupados por semelhança, as quais representaram as temáticas mais significativas da análise, que serão detalhadas e discutidas a seguir.

\section{RESULTADOS}

A Tabela 1 apresenta a distribuição da combinação da descritores e palavra-chave.

Tabela 1 - Distribuição dos artigos segundo combinação de palavra-chave e descritores (2000-2013). Santa Maria, RS, 2014.

\begin{tabular}{lccc}
\hline DESCRITORES E PALAVRA-CHAVE & LILACS & SCIELO & PUBMED \\
\hline trabalho noturno and cronotipo and enfermagem & 1 & 0 & - \\
night work and crhonotype and nursing & - & - & 5 \\
trabalho em turnos and cronotipo and enfermagem & 4 & 4 & - \\
shift work and crhonotype and nursing & - & - & 7 \\
ritmo circadiano and cronotipo and enfermagem & 3 & 3 & - \\
circadian Rhythm and crhonotype and nursing & - & - & 7 \\
\hline
\end{tabular}

Após a leitura do resumo dos 34 estudos encontrados, foram excluídas as repetições (16), tese (1), protocolo de pesquisa (3), revisão da literatura (1), estudo anterior ao período selecionado (1) e estudo que não respondia a questão norteadora (1), sendo selecionados 11 artigos que se adequaram aos critérios de inclusão. Acrescentaramse a esta amostra estudos selecionados intencionalmente em arquivos pessoais das autoras e outros, obtidos a partir de referências dos artigos incluídos na busca sistematizada, estratégia indicada por autores ${ }^{7}$, totalizando uma amostra de 14 estudos.

As pesquisas utilizaram como instrumento de coleta de dados, predominantemente, o Questionário de Matutinidade e Vespertinidade de Horne, Östberg $(92,86 \%)$, o que sugere que esse instrumento é o que mais se tem utilizado para identificar 


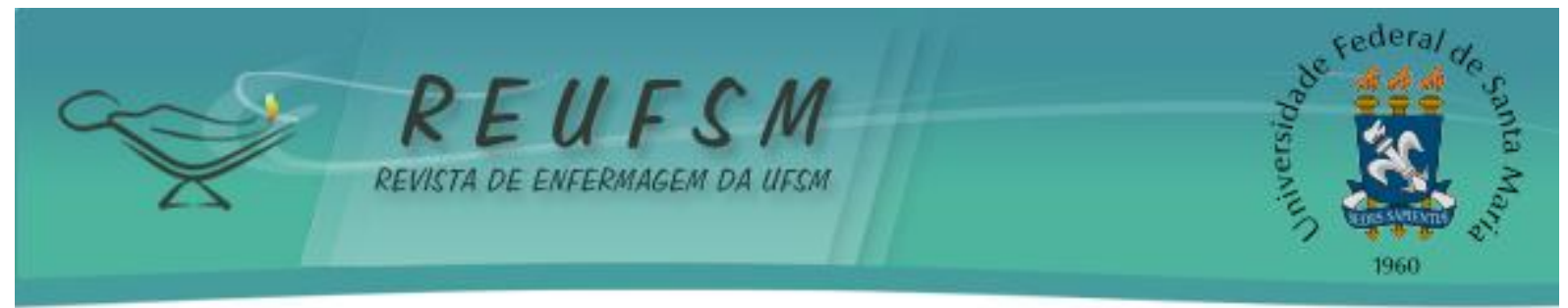

o cronotipo em trabalhadores de enfermagem. Os participantes dos estudos selecionados foram enfermeiros $(42,86 \%, n=6)$, profissionais de enfermagem $(35,71 \%, n=5)$ e enfermeiros-docentes $(7,14 \%, n=1)$ conforme está apresentado no Quadro 1:

\begin{tabular}{|c|c|c|}
\hline PERIÓDICO & TIPO DE ESTUDO E OBJETIVO & PRINCIPAIS RESULTADOS \\
\hline $\begin{array}{l}\text { Revista de } \\
\text { Ciências } \\
\text { Médicas - } \\
\text { 2001. }^{8}\end{array}$ & $\begin{array}{l}\text { Caracterizar um grupo de enfermeiros } \\
\text { de dois hospitais de ensino quanto ao } \\
\text { cronotipo e identificar possíveis } \\
\text { associações entre cronotipo, turno de } \\
\text { trabalho e idade. }\end{array}$ & $\begin{array}{l}\text { Houve predomínio do cronótipo } \\
\text { indiferente. A maioria dos enfermeiros } \\
\text { moderadamente matutinos trabalhavam } \\
\text { no turno da manhã, sugerindo } \\
\text { adequação entre o turno e cronotipo. }\end{array}$ \\
\hline $\begin{array}{l}\text { Nursing (São } \\
\text { Paulo) - 2001.9 }\end{array}$ & $\begin{array}{l}\text { ldentificar } 0 \quad \text { cronotipo } \\
\text { enfermeiras-docentes. }\end{array}$ & $\begin{array}{l}\text { Houve predomínio do cronotipo } \\
\text { matutino. Os autores concluíram que as } \\
\text { preferências individuais foram ao } \\
\text { encontro do atual regime de trabalho } \\
\text { dos pesquisados. }\end{array}$ \\
\hline $\begin{array}{c}\text { Revista da } \\
\text { Escola de } \\
\text { Enfermagem da } \\
\text { USP - 2004. }\end{array}$ & $\begin{array}{l}\text { Identificar os cronotipo dos } \\
\text { enfermeiros dos diferentes turnos, } \\
\text { estudar os padrões de sono e } \\
\text { identificar os níveis de ansiedade- } \\
\text { estado e ansiedade-traço. }\end{array}$ & $\begin{array}{l}\text { O cronotipo predominante dos } \\
\text { enfermeiros do turno maturino foi o } \\
\text { matutino moderado e os do vespertino e } \\
\text { noturno foi o indiferente. Os } \\
\text { enfermeiros do turno matutino dormem } \\
\text { menos, acordam mais cedo e com a } \\
\text { ajuda do despertador em relação aos } \\
\text { outros turnos. }\end{array}$ \\
\hline $\begin{array}{l}\text { Journal } \\
\text { Professional } \\
\text { Nursing - } \\
\text { 2004. }^{11}\end{array}$ & $\begin{array}{l}\text { Avaliar as percepções sobre } 0 \\
\text { cronotipo, flexibilidade de sono e } \\
\text { estilo de vida de trabalhadores de } \\
\text { enfermagem e seus companheiros. }\end{array}$ & $\begin{array}{l}\text { Os resultados indicam que os } \\
\text { trabalhadores que atuam em turnos e } \\
\text { seus parceiros são congruentes em suas } \\
\text { percepções sobre o impacto do trabalho } \\
\text { em turnos. }\end{array}$ \\
\hline $\begin{array}{l}\text { * Revista de } \\
\text { Ciências } \\
\text { Médicas - } \\
\text { 2004. }{ }^{12}\end{array}$ & $\begin{array}{l}\text { Classificar o cronotipo de alunos } \\
\text { universitários. }\end{array}$ & $\begin{array}{l}\text { Houve predomínio do cronotipo } \\
\text { indiferente entre os pesquisados. }\end{array}$ \\
\hline $\begin{array}{c}\text { Revista } \\
\text { Brasileira de } \\
\text { Enfermagem- } \\
\text { 2005. }\end{array}$ & $\begin{array}{l}\text { Estudo exploratório descritivo com o } \\
\text { objetivo de caracterizar o padrão do } \\
\text { ciclo vigília-sono de estudantes } \\
\text { universitários no início do primeiro } \\
\text { ano da graduação. }\end{array}$ & $\begin{array}{l}\text { Em uma primeira etapa do estudo, } \\
\text { identificou-se que os indivíduos } \\
\text { adotavam horários mais condizentes } \\
\text { com seu cronotipo; na segunda etapa } \\
\text { não foram detectadas diferenças } \\
\text { significativas o que indica a sujeição dos } \\
\text { sujeitos aos horários impostos pelo } \\
\text { desenvolvimento das atividades } \\
\text { acadêmicas em detrimento de suas } \\
\text { preferências individuais. }\end{array}$ \\
\hline $\begin{array}{l}{ }^{*} \text { ConScientiae } \\
\text { Saúde - 2008. }{ }^{14}\end{array}$ & $\begin{array}{l}\text { Identificar o cronotipo de profissionais } \\
\text { da saúde e verificar se seus turnos de } \\
\text { trabalho correspondiam ou não ao } \\
\text { período em que estariam atuando. }\end{array}$ & $\begin{array}{l}\text { Houve predomínio do cronótipo } \\
\text { indiferente. Uma parcela dos } \\
\text { pesquisados classificados como } \\
\text { moderadamente matutinos não } \\
\text { apresentava adequação entre turno de } \\
\text { trabalho e cronótipo. }\end{array}$ \\
\hline
\end{tabular}




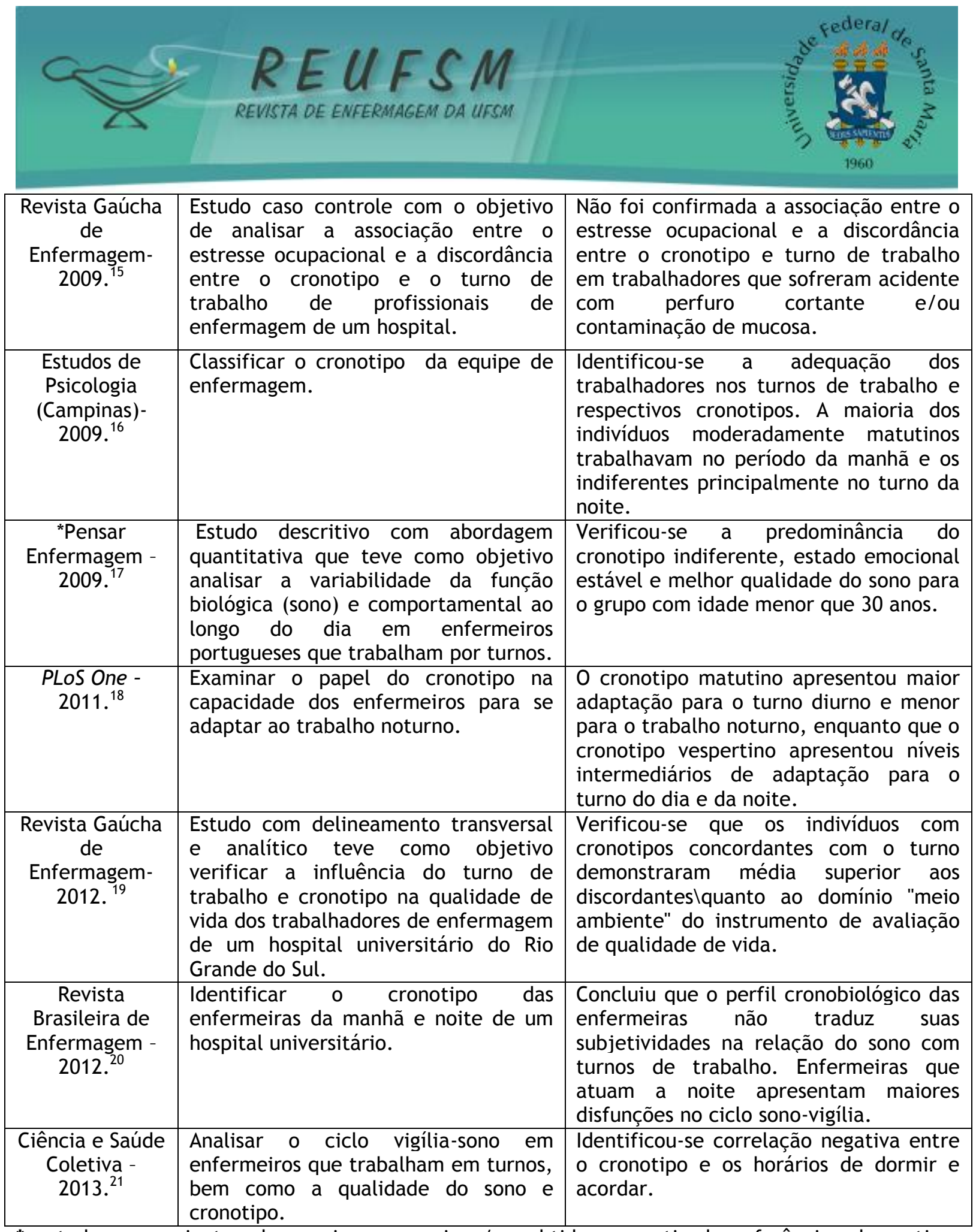

* estudos provenientes de arquivos pessoais e/ou obtidos a partir de referências dos artigos incluídos na busca sistematizada.

Quadro 1- Artigos selecionados para integrar a revisão. Santa Maria, RS, 2014.

Por meio da análise dos artigos percebe-se que os estudos versavam sobre duas temáticas: relação do cronotipo e turno de trabalho e associação do cronotipo e variáveis.

\section{Relação do cronotipo e turno de trabalho}

De acordo com a classificação do cronotipo, observou-se a predominância do cronotipo indiferente. ${ }^{8,12,14-17,21}$ Esse dado é positivo uma vez que este cronotipo está 


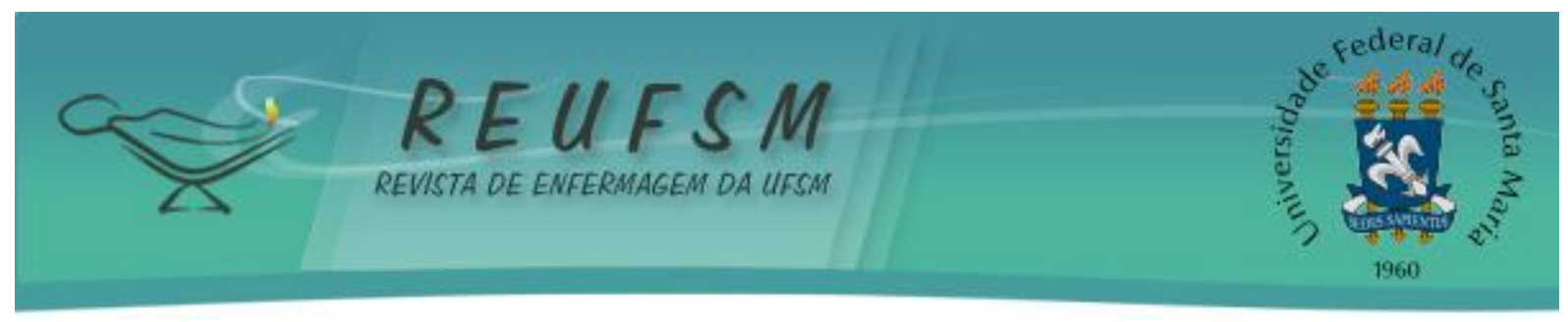

adequado a qualquer turno de trabalho, o que poderá repercutir na otimização da assistência prestada ${ }^{20}$, e na saúde do trabalhador.

Salienta-se que a realização de atividades em período divergente ao cronotipo pode implicar em dificuldades de adaptação para o organismo. ${ }^{4,20}$ Estudo realizado com trabalhadores da equipe de enfermagem de um hospital universitário do Sul do Brasil constatou que $70,58 \%$ estavam alocados de forma discordante com relação ao cronotipo e turno de trabalho. ${ }^{19}$

Já a concordância do cronotipo com o turno de trabalho é fator impactante nos serviços de saúde, pois pode contribuir para o bem-estar do profissional, satisfação profissional e qualidade do cuidado em saúde. Em pesquisas realizadas com trabalhadores de enfermagem foi identificado que a maioria das pessoas estavam adequadas ao turno de trabalho e cronotipo..$^{8-10,15-16}$

Estudo realizado com 14 enfermeiros docentes de um colégio técnico identificou que as preferências individuais foram ao encontro do atual regime de trabalho dos pesquisados, sendo que estes haviam optado pelo período de trabalho conforme 0 cronotipo. ${ }^{9}$

Deve-se considerar que a exposição do indivíduo a situações desconfortáveis e ambientes perigosos potencializa a ocorrência de acidentes de trabalho e o desenvolvimento de doenças ${ }^{15}$, sendo que a alocação de um trabalhador em turno divergente ao seu cronotipo pode sugerir alterações na saúde.

\section{Associação do cronotipo e variáveis}

Quando associado o cronotipo e turno de trabalho de enfermeiros de duas instituições hospitalares de ensino, evidenciou-se que os indivíduos moderadamente matutinos trabalhavam na sua maioria no período da manhã $(p<0,005)$ e os indiferentes principalmente no turno da tarde $(p<0,005)$. Não houve associação entre cronotipo e idade. ${ }^{8}$

Estudo realizado com enfermeiros de um hospital de clínicas não encontrou diferença significativa entre os níveis de ansiedade-traço entre os turnos de trabalho, embora a maioria dos indivíduos estivesse alocada em turno correspondente ao cronotipo. ${ }^{9}$ Pesquisa conduzida com a equipe de enfermagem identificou que $75,7 \%$ dos trabalhadores estavam adequados com relação ao cronotipo e turno de trabalho (maioria com cronotipo indiferente), sendo identificado sintomas de stress em $55,2 \%$ dos indivíduos. ${ }^{16}$

Pesquisa realizada com profissionais de enfermagem (enfermeiros, técnicos de enfermagem e auxiliares de enfermagem) de um hospital da região Sul do Brasil não encontrou evidencia de associação entre o cronotipo e qualidade de vida. ${ }^{19}$

Investigação realizada em um hospital de clínicas com profissionais de enfermagem que sofreram acidente com perfuro cortante e/ou de contaminação de mucosa não confirmou associação entre o estresse ocupacional e a discordância entre o cronotipo e o turno de trabalho. ${ }^{15}$ Estudo realizado com estudantes universitários de cursos da área de ciências exatas e ciências biológicas, em que foram associados o Índice de Qualidade de Sono de Pittsburgh e o Questionário de Matutinidade/Vespertinidade, não encontrou diferença estatística significativa entre os padrões habituais de sono e o cronotipo em duas etapas de coleta de dados (Etapa 1 e 2, $p>0,05){ }^{13}$

Pesquisa com o objetivo de examinar o papel do cronotipo na capacidade de adaptação de enfermeiros americanos ao trabalho noturno identificou que os que atuavam neste turno apresentaram menor adaptação ao horário de trabalho em relação aos enfermeiros atuantes no turno diurno $(p<0,01)$. 0 cronotipo matutino apresentou maior adaptação para o turno diurno e menor para o trabalho noturno, enquanto que o cronotipo 


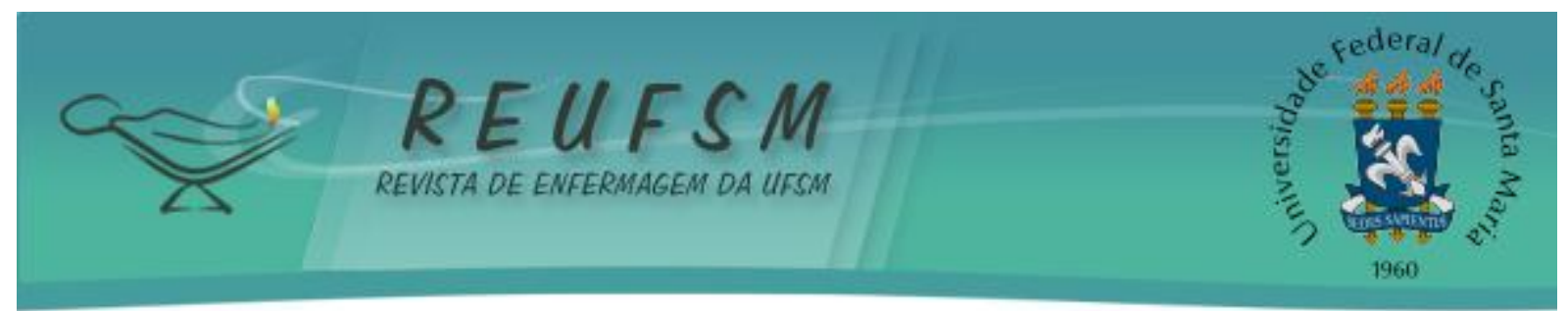

vespertino apresentou níveis intermediários de adaptação para o turno do dia e da noite. Os autores concluem que o cronotipo influencia a eficácia da adaptação em turnos. ${ }^{18}$

Os trabalhadores que atuam no noturno experimentam uma série de situações que comprometem o descanso diurno, como os micro dispertares, sons e interferências do meio ambiente e a luz solar ${ }^{22}$, o que se reflete na saúde do trabalhador.

Pesquisa que analisou a qualidade do sono e o cronotipo de enfermeiras portuguesas encontrou correlação negativa entre o cronotipo e os horários de dormir e acordar, o que sugere que pessoas com altos escores para cronotipo (tendência a matutinidade) mostram maior disposição para dormir mais cedo e acordar mais cedo; enquanto que pessoas com baixos escores para cronotipo (tendência a vespertinidade) mostram maior disposição para dormir tarde e acordar tarde. ${ }^{21}$

Estudo conduzido com enfermeiros de dois hospitais públicos localizados na Austrália identificou que os trabalhadores que atuam em turnos e seus companheiros possuem semelhança nas suas percepções sobre o impacto do trabalho em turnos. Foi encontrada associação entre cronotipo e sono/fadiga no turno diurno $(p<0,05)$, sugerindo que os trabalhadores com cronotipo vespertino percebem mais problemas relacionados ao sono/cansaço. ${ }^{11}$

Pesquisa realizada junto a trabalhadores de enfermagem que atuavam em Unidades de Tratamento Intensivo identificou que 63,2\% automedicavam-se, sendo os analgésicos e os antiinflamatórios os mais citados, o que sugere adoecimento dessas pessoas. ${ }^{23}$ Para promover a saúde dos profissionais que atuam em turnos, uma possível estratégia seria sensibilizá-los sobre as situações implicadas no processo de trabalho que os tornam vulneráveis ao adoecimento.

No cenário do trabalho em saúde, a forma como está organizado o trabalho somado as cargas e responsabilidades causam desgaste nos trabalhadores ${ }^{24}$, que muitas vezes negligenciam o cuidado em direção ao seu próprio estado de saúde. ${ }^{25}$

Assim, embora haja a suposição de que a discordância entre cronotipo e turno de trabalho tenha interferência na saúde do trabalhador, outras causas relacionadas ao contexto do trabalho precisam ser consideradas nessa avaliação. Portanto, considerar o conhecimento sobre o cronotipo se constitui em um dado importante na medida em que poderá repercutir na qualidade da assistência.

\section{CONCLUSÕES}

A partir dos dados encontrados, pode-se concluir que a produção do conhecimento sobre o cronotipo na enfermagem é incipiente. Contudo, é possível constatar que há preocupação de alguns pesquisadores sobre o desenvolvimento dessa temática, sendo encontradas pesquisas que identificaram o cronotipo dos trabalhadores, associando-o ao turno de trabalho como qualidade do sono e capacidade de adaptação ao turno, o que demonstra que o cronotipo pode influenciar a saúde do trabalhador de enfermagem que atua em turnos.

No entanto, embora tenham sido encontradas influências do cronotipo na saúde do trabalhador, são necessárias investigações com outras variáveis com o objetivo de identificar possíveis interferências da alocação de um trabalhador em turno discordante ao cronotipo.

Assim, no contexto da saúde, são necessárias ações que busquem alocar o trabalhador no turno de sua preferência, de acordo com seu cronotipo, na perspectiva de minimizar as possíveis repercussões, pois as consequências não se limitam aos prejuízos causados ao trabalhador, mas também a assistência prestada. 


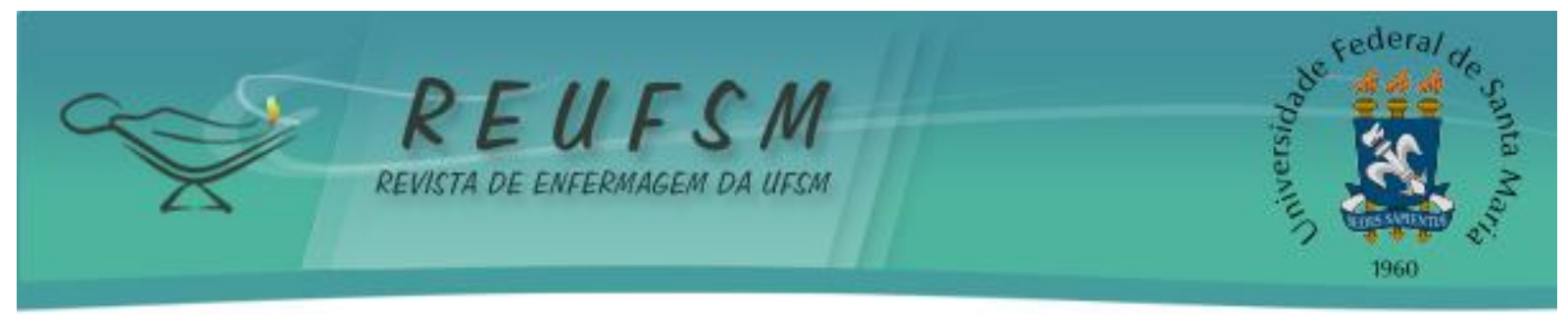

\section{REFERÊNCIAS}

1. Silva RM, Beck CLC, Zeitoune RCG, Prestes FC, Tavares JP, Guerra ST. Sentido do trabalho para enfermeiros noturnos de um hospital universitário: estudo descritivo. Online Braz J Nurs [Internet]. 2011 [acesso em 2014 maio 25];10(3). Disponível em: http://www.objnursing.uff.br/index.php/nursing/article/view/3433/1089.

2. Mohamed N, Gabr H. Develop accreditation standards for nursing departments at Mansoura University Hospital. J Educ Practice. 2013;4(7):37-48.

3. Jonason PK, Jones A, Lyons M. Creatures of the night: chronotypes and the dark triad traits. Pers Individ Dif. 2013;55(5):538-41.

4. Horne JA, Ostberg 0 . A self-assesment questionnaire to determine morningnesseveningness in human circadian rhythms. Int Journ Chrobiol. 1976;4(2):97-110.

5. Mendes KDS, Silveira RCCP, Galvão CM. Revisão integrativa: método de pesquisa para a incorporação de evidências na saúde e na enfermagem. Texto \& Contexto Enferm. 2008;17(4):758-64.

6. Crossetti MGO. Revisão integrativa de pesquisa na enfermagem o rigor cientifico que the é exigido. Rev Gaúch Enferm. 2012;33(2):8-9.

7. Whittemore R, Knalf $\mathrm{K}$. The integrative review: updated methodology. J Adv Nurs. 2005;52(5):546-53.

8. De Martino MMF, Ceolim MF. Avaliação do cronótipo de um grupo de enfermeiros de hospitais de ensino. Rev Cienc Med. 2001;10(1):19-27.

9. Campos MLP, De Martino MMF. Estudos das características cronobiológicas dos enfermeiros docentes: cronótipo. Nursing (São Paulo). 2001;4(42):31-4.

10. Campos MLP, De Martino MMF. Aspectos cronobiológicos do ciclo vigília-sono e níveis de ansiedade dos enfermeiros nos diferentes turnos de trabalho. Rev Esc Enferm USP. 2004;38(4):415-21.

11. Newey CA, Hood BM. Determinants of shift-work adjustment for nursing staff: the critical experience of partners. J Prof Nurs. 2004;20(3):187-95.

12. De Martino MMF, Ling SY. Características cronobiológicas de um grupo de alunos universitários de enfermagem. Rev Cienc Med. 2004;13(1):43-9.

13. Furlani R, Ceolim MF. Padrões de sono de estudantes ingressantes na graduação em enfermagem. Rev Bras Enferm. 2005;58(3):320-4.

14. Alves AL, Campos FP, Deghi LP, Gamberini MC. Identificação do cronótipo de membros de uma equipe de enfermagem que trabalham nos turnos diurno e noturno. Conscientiae Saúde. 2008;7(1):49-54.

15. Dalarosa MG, Lautert L. Acidente com material biológico no trabalhador de enfermagem em um hospital de ensino: estudo caso-controle. Rev Gaúch Enferm. 2009;30(1):19-26.

16. Ferreira LRC, De Martino MMF. Stress no cotidiano da equipe de enfermagem e sua correlação com o cronótipo. Estud psicol (Campinas). 2009;26(1):65-72.

17. De Martino MMF, Basto ML. Qualidade do sono, cronótipos e estados emocionais: o caso de enfermeiros portugueses que trabalham por turnos. Pensar Enfermagem. 2009;13(1):49-60. 


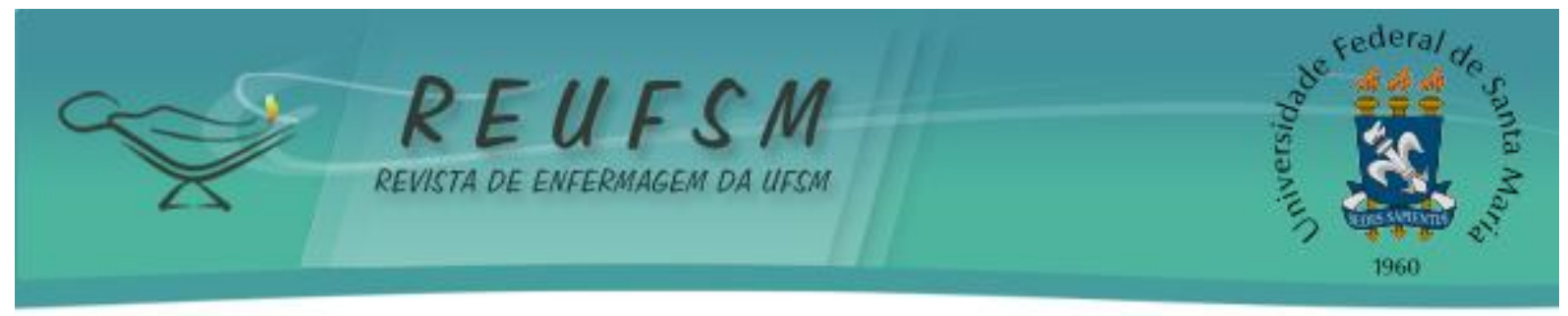

18. Gamble KL, Motsinger-Reif AA, Hida A, Borsetti HM, Servick SV, Ciarleglio CM, et al. Shift work in nurses: contribution of phenotypes and genotypes to adaptation. PLoS One. 2011;6(4):1-12.

19. Souza SBC, Tavares JP, Macedo ABT, Moreira PW, Lautert L. Influência do turno de trabalho e cronotipo na qualidade de vida dos trabalhadores de enfermagem. Rev Gaúch Enferm. 2012;33(4):79-85.

20. Xavier KGS, Vaghetti $\mathrm{HH}$. Aspectos cronobiológicos do sono de enfermeiras de um hospital universitário. Rev Bras Enferm. 2012;65(1):135-40.

21. De Martino MMF, Abreu ACB, Barbosa MFS, Teixeira JEM. The relationship between shift work and sleep patterns in nurses. Ciênc Saúde Coletiva. 2013;18(3):763-8.

22. De Martino MMF. Arquitetura do sono diurno e ciclo vigília-sono em enfermeiros nos turnos de trabalho. Rev Esc Enferm USP. 2009;43(1):194-9.

23. Vieira TG, Beck CLC, Dissen CM, Camponogara S, Gobatto M, Coelho APF. Adoecimento e uso de medicamentos psicoativos entre trabalhadores de enfermagem de unidades de terapia intensiva. Rev Enferm UFSM [Internet]. 2013 [acesso em 2014 maio 6];3(2):205-14. Disponível em: http://cascavel.ufsm.br/revistas/ojs-

2.2.2/index.php/reufsm/article/view/7538/pdf.

24. Guido LA, Silva RM, Goulart CT, Kleinübing RE, Umann J. Estresse e coping entre enfermeiros de unidade cirúrgica de hospital universitário. Rev RENE. 2012;13(2):428-36.

25. Neves MJAO, Branquinho NCSS, Paranaguá TTB, Barbosa MA, Siqueira KM. Influência do trabalho noturno na qualidade de vida do enfermeiro. Rev Enferm UERJ. 2010;18(1):42-7.

Data de recebimento: $11 / 02 / 2014$

Data de aceite: 29/10/2014

Contato com autor responsável: Rosângela Marion da Silva

Endereço: Avenida Roraima, $\mathrm{n}^{\circ} 1000$, Centro de Ciências da Saúde, $3^{\circ}$ andar, sala $1305^{\mathrm{a}}$, Cidade Universitária, Bairro Camobi, Santa Maria, RS, CEP: 97105-900

E-mail: cucasma@terra.com.br 